\title{
ALMA and solar research
}

\author{
Marian Karlický and Miroslav Bárta \\ Astronomical Institute of the Academy of Sciences of the Czech Republic, \\ CZ - 25165 Ondřejov, Czech Republic \\ email: karlicky@asu.cas.cz, barta@asu.cas.cz
}

\begin{abstract}
The ALMA (Atacama Large Millimeter/sub-millimeter Array) is the large interferometer that will consist up to 64 high-precision antennas operating in the $31.3-950 \mathrm{GHz}$ frequency range. In this range unique observations in cosmology, cold universe, galaxies, stars and their formations, and so on are expected. Among these objectives there is a unique possibility to observe the Sun and to address outstanding issues of solar physics. The ALMA is shortly described and then the new ESO-ALMA European node (ARC) built at Ondřejov Observatory is presented. The new ARC is the only one in Europe oriented to solar physics. The requirements and limitations for ALMA solar observations, as well as some examples of possible solar-oriented ALMA projects, are mentioned.
\end{abstract}

Keywords. instrumentation: high angular resolution, interferometers, Sun: radio radiation

\section{Introduction}

The Atacama Large Millimeter/sub-millimeter Array (www.almaobservatory.org) is a worldwide project to construct a large interferometer (up to 64 high-precision $12 \mathrm{~m}$ and $7 \mathrm{~m}$ antennas, operating in the $31.3-950 \mathrm{GHz}$ frequency range) in Chajnantor plain of the Chilean Andes at the altitude $5000 \mathrm{~m}$ above sea level. The telescope will be operated from the nearby operational center located at the elevation $2900 \mathrm{~m}$. Four main centers are built for the preparation of proposals for observations, data distribution, data analysis and ALMA science and technical supports. Besides the center in Santiago de Chile, there is main European ESO-ALMA center in Garching, Germany (www.eso.org/sci/facilities/alma/arc/), North American ALMA Science Center (NAASC) in Charlottesville, Virginia, USA (science.nrao.edu/alma/index.shtml), and East-Asian ALMA Regional Center in Mitaka, Japan (alma.mtk.nao.ac.jp/ EA-ARC/). The European ALMA Regional Center (ARC) is, besides the main center in Garching, formed by a distributed structure of seven additional nodes (ARC nodes) which cover various branches of expected ALMA science and provide better geographical accessibility for ALMA users in Europe.

The science program of ALMA is very broad. It includes molecular clouds in the cold universe, cosmology, the formation of galaxies and clusters of galaxies, formation of stars and planets, astrochemistry, comets and also the solar research. The solar physics was included into this ambitious project primarily thanks to A. O. Benz, T. Bastian, S. White, M. Kundu and others. There are several papers advertising ALMA as a future observing instrument for solar research, e.g Bastian (2002) and Loukitcheva et al. (2008).

In this paper, we want to inform about the present status of the ALMA project, about the new ESO-ALMA node at Ondřejov Observatory which is oriented mainly to solar physics, and about a unique potential of ALMA for the solar physics research. 
Table 1. ALMA parameters. $\lambda_{\mathrm{mm}}$ is the wavelength in $\mathrm{mm}$.

\begin{tabular}{ll}
\hline Antennas & 64 \\
& $\left(25 \mu \mathrm{m} \mathrm{rms}, 0.6^{\prime \prime}\right.$ pointing $)$ \\
Collecting Area & $>7000 \mathrm{~m}^{2}$ \\
Receivers & 10 bands in $31.3-950 \mathrm{GHz}$ \\
& $0.3-9.6 \mathrm{~mm}$ \\
Field of view & $21^{\prime \prime} \times \lambda_{\mathrm{mm}}$ \\
Spatial resolution & $0.02^{\prime \prime} \times \lambda_{\mathrm{mm}} \times(10 \mathrm{~km} /$ baseline $)$ \\
Number of baselines & up to 2016 \\
\hline
\end{tabular}

\section{ALMA}

ALMA construction started in 2002 and the project will be completed in 2012. The telescope is located in the Atacama desert in Chile, at the elevation of $5000 \mathrm{~m}$, where superior transmission properties of the atmosphere at $\mathrm{mm} / \mathrm{sub}-\mathrm{mm}$ wavelengths are found. The first call for proposals of observations is expected to be released at the beginning of 2011.

ALMA is a Fourier synthesis telescope. It will consist up to 64, $12 \mathrm{~m}$ and $7 \mathrm{~m}$ antennas (for ALMA parameters, see Table 1). Each pair of antennas measures one Fourier component of the brightness distribution of the radio source. An image of the source is then computed by the inverse Fourier transform of all $N(N-1) / 2$ components, where $N$ is the number of antennas in the array, i.e. 2016 in ALMA case. The instantaneous field of view of the instrument is given by the antenna size $(12 \mathrm{~m})$ and scales linearly with the wavelength (see Table 1). The spatial resolution is determined by the maximum separation of antennas (up to $14 \mathrm{~km}$ ) and for its wavelength scaling see Table 1. ALMA is also designed to perform very sensitive spectral line observations in the $31.3-950 \mathrm{GHz}$ frequency range, which will be divided into 10 frequency bands. For more details about ALMA, see www.almaobservatory.org.

Proposals for ALMA observations will be prepared by the ALMA Observing Tool software. This will be a two-step procedure, where in the first step the scientific objectives and goals are considered, and if approved by the scientific committee then, in the second step, detailed technical specifications are checked. On the other hand, observed data will be reduced and analyzed by the Common Astronomy Software Applications (CASA) (casa.nrao.edu). Now, both these software packages are in a final testing phase.

\section{ESO-ALMA node (ARC) in Ondřejov}

In November 2008, we have prepared and presented to ESO a proposal to build a new ESO-ALMA node (ARC) in Ondřejov. In December 2009 the ESO-ALMA node at the Astronomical Institute of the Academy of Sciences was formally approved by the ESO Director. The main purpose of the European ARC nodes is to provide on-line as well as face-to-face support to the ALMA users in matters of proposal preparation, observation planning, and data reduction. The newly formed node in Ondřejov will provide scientific and technical support mainly in the field of solar physics, but also in galactic/extragalactic and relativistic astrophysics, and laboratory measurements and quantum-physics modelling of molecular spectral lines (in cooperation with the Institute of Chemical Technology in Prague). Since it is a unique node providing support in solar research with ALMA in Europe it is, according to the ARC strategy, open to all European ALMA users requiring assistance with accomplishing their solar-oriented ALMA project. Formation of the new node in Ondřejov also improved geographical 
distribution of European ARC structure. Thus, namely for topics which are covered also by other nodes (e.g. galactic physics), it represents a natural regional center for support of users from Central and Eastern Europe. For details about the new ESO-ALMA node, see http://www. asu.cas.cz/alma. Present activities of the team of this node are: a) preparation of the infrastructure of the ARC node (computers, data servers, fast internet connections), b) participation in official tests of CASA and ALMA Observing Tool softwares, c) communication with other European ESO-ALMA nodes through telephone conferences and face-to-face meetings and d) presentation of lectures about ALMA for students.

\section{ALMA and solar research}

In the following the limitations of ALMA and requirements for solar observations are summarized:

- Field of view (FOV) of ALMA is rather small (Table 1). To increase the FOV, an observing technique called "on-the-fly" has to be used (Bastian, 2002).

- Comparing with other astrophysical radio sources the solar radio flux is very strong. Therefore, an appropriate attenuation of the signal is necessary.

- It will be good to scatter visible/IR part of the spectrum by the milling of the surface of antennas.

- An advanced calibration technique is necessary.

- For transient phenomena such as solar flares, a flexible communication between scientists and observing staff regarding observed targets is necessary.

Examples of possible ALMA solar-physics studies follow:

- Study of the quiet chromosphere

- Study of prominences and filaments

- Study of solar radio recombination lines

- Study of chromospheric oscillations and waves

- Study of microjets in sunspot penumbrae

- Study of solar flares

\section{Concluding remarks}

ALMA is designed to observe many objects in the Universe. Since the observing capabilities of ALMA are advanced, an involvement of the solar community in ALMA is highly desirable. To encourage potential solar observers, the new ESO-ALMA node (ARC) at Ondřejov Observatory is being built under the supervision of ESO. Although we are aware of problems which are specific for solar observations (small field of view, strong radio flux, calibration and so on), we hope that the advanced techniques will overcome these difficulties.

\section{Acknowledgements}

The authors are indebted to Prof. T. Wilson and Dr. P. Andreani from ESO for their great support in building the Ondrejov ALMA node. This paper was supported by Grant 300030701 of the Grant Agency of the Academy of Sciences of the Czech Republic.

\section{References}

Bastian, T. S. 2002, Astron. Nachr., 323, 271

Loukitcheva, M. A., Solanki, S. K., \& White, S. 2008, Astrophys. Space Sci., 313, 197 